\title{
Super-resolution fluorescence imaging of intracellular mutant huntingtin protein reveals a population of fibrillar aggregates co-existing with compact perinuclear inclusion bodies
}

\author{
Steffen J Sahl $1^{1 *}$, Lucien E Weiss ${ }^{1}$, Lana Lau', Judith Frydman², WE Moerner ${ }^{1}$ \\ From Molecular Neurodegeneration: Basic biology and disease pathways \\ Cannes, France. 10-12 September 2013
}

\section{Background}

The identities of toxic aggregate species in Huntington's disease (HD) pathogenesis remain unclear. While polyQ-expanded mutant huntingtin $(\mathrm{Htt})$ is known to accumulate in compact inclusion bodies inside neurons, this is widely thought to be a protective coping response that sequesters misfolded conformations or aggregated states of the mutated protein.

\section{Materials and methods, results}

To define the spatial distributions of fluorescentlylabeled Htt-exon1 species in the cell model PC12m (terminally differentiated into sympathetic-neuron-like cells with nerve growth factor), we employed highly sensitive single-molecule-based and stimulated emission depletion (STED) super-resolution fluorescence imaging modalities. In addition to inclusion bodies and the diffuse pool of monomers and oligomers, fibrillar aggregates $\sim 100 \mathrm{~nm}$ in diameter and up to $\sim 1-2 \mu \mathrm{m}$ in length were observed for pathogenic polyQ tracts (expression experiments with 46 and 97 repeats) after targeted photo-bleaching of the inclusion bodies [1]. These short structures bear a striking resemblance to fibers described in vitro [2]. We identified a sharp cutoff behavior of maximum fibril length and documented the ensuing bundling of these fibers into denser arrangements of varying complexity, both in the cytosolic space and inside the neuritic processes.

\section{Conclusions}

Definition of the diverse $\mathrm{Htt}$ structures in cells will provide an avenue to link the impact of pharmacological agents to aggregate populations and morphologies. The latest observations w.r.t. co-localization of $\mathrm{Htt}$ with various quality control proteins such as the chaperone Hsp70 will be presented.

\section{Authors' details \\ 'Department of Chemistry, Stanford University, Stanford, CA, USA. \\ ${ }^{2}$ Department of Biology Stanford University, Stanford, CA, USA.}

\section{Published: 13 September 2013}

\section{References}

1. Sahl SJ, Weiss LE, Duim WC, Frydman J, Moerner WE: Cellular inclusion bodies of mutant huntingtin exon 1 obscure small fibrillar aggregate species. Sci Rep 2012, 2:895.

2. Duim WC, Chen B, Frydman J, Moerner WE: Sub-diffraction imaging of huntingtin protein aggregates by fluorescence blink-microscopy and atomic force microscopy. ChemPhysChem 2011, 12:2387-2390.

doi:10.1186/1750-1326-8-S1-018

Cite this article as: Sahl et al:: Super-resolution fluorescence imaging of intracellular mutant huntingtin protein reveals a population of fibrillar aggregates co-existing with compact perinuclear inclusion bodies. Molecular Neurodegeneration 2013 8(Suppl 1):018. 